Valcárcel-Linares, D.; Torres-Lacomba, M. (2020). Pain in Competitive Karate during Training. Prevalence Study. Revista Internacional de Medicina y Ciencias de la Actividad Física y el Deporte vol. 20 (77) pp. 335-352 Http://cdeporte.rediris.es/revista/revista78/artdolor1145.htm

\title{
ORIGINAL
}

\section{DOLOR EN EL ENTRENAMIENTO EN EL KARATE DE COMPETICIÓN. ESTUDIO DE PREVALENCIA}

\section{PAIN IN COMPETITIVE KARATE DURING TRAINING. PREVALENCE STUDY}

\author{
Valcárcel-Linares, D. ${ }^{1}$ y Torres-Lacomba, M. $^{2}$ \\ 1 Graduado en Fisioterapia. Universidad de Alcalá, Madrid (España) \\ david.valcarcel.1994@gmail.com \\ 2 Doctora en Biomedicina. Profesora titular de Universidad. Coordinadora del Grupo de \\ Investigación Fisioterapia en los Procesos de la Salud de la Mujer. Universidad de Alcalá, Madrid \\ (España) maria.torres@uah.es
}

AGRADECIMIENTOS: a mi familia, a Carlos Gutiérrez Ortega, a mis amigos, a mis compañeros de clase, a mi sensei, a todos los karatekas y participantes del estudio, porque sin vosotros esto no sería posible.

Código UNESCO / UNESCO code: 3213.11 Fisioterapia / Physiotherapy. Clasificación Consejo de Europa / Council of Europe classification: 14. Fisioterapia y rehabilitación / Physiotherapy and rehabilitation

Recibido 18 de junio de 2018 Received June 18, 2018

Aceptado 22 de junio de 2019 Accepted June 22, 2019

\section{RESUMEN}

Objetivos: conocer la prevalencia de dolor derivada del entrenamiento en karatekas de competición y su relación con los factores antropométricos y deportivos. Métodos: Estudio de prevalencia. Un total de 56 karatekas cumplimentaron un cuestionario al finalizar su entrenamiento de karate. Resultados: La prevalencia del dolor ha sido del $67.9 \%$. Se ha demostrado que la rodilla es la zona más prevalente en relación con el dolor (31\%) y con la sensación subjetiva de inestabilidad $(50 \%)$. Se han encontrado relaciones estadísticamente significativas entre el dolor y la edad $(p=0.04)$, el Índice de Masa Corporal $(p=0.045)$, el grado de cinturón $(p=0.017)$ y la frecuencia de entrenamiento de karate $(p=0.011)$. Conclusiones: el dolor en karatekas de competición durante los entrenamientos muestra una alta prevalencia en la muestra estudiada. Hubo una correlación estadísticamente significativa en el dolor en los karatekas con un mayor índice de masa corporal, mayor edad, mayor grado de cinturón y mayor frecuencia de entrenamiento. 
PALABRAS CLAVE: artes marciales, prevalencia, dolor, inestabilidad articular.

\begin{abstract}
Objectives: to find out the pain prevalence in competitive karate practitioners derived from training and its relation to anthropometric and sports factors.

Methods: prevalence study. A total of 56 karate practitioners completed a questionnaire at the end of their karate training. Results: the pain prevalence was $67.9 \%$. The knee has been shown to be the most prevalence zone related to pain $(31 \%)$ as well as related to instability subjective feeling (50\%). There were significant statistical correlations between pain and age $(p=0.04)$, Body Mass Index $(p=0.045)$, belt level $(p=0.017)$ and karate training frequency $(p=0.011)$. Conclusions: the pain in competitive karate practitioners during training shows a high prevalence in the sample studied. There was a statistically significant correlation in pain in karate practitioners with higher body mass index, higher age, higher belt level and higher karate training frequency.
\end{abstract}

KEYWORDS: martial arts, prevalence, pain, joint instability.

\title{
1. INTRODUCCIÓN
}

El karate, cuyo significado es "mano vacía", es un arte marcial desarrollado en Okinawa (Japón). Existen dos modalidades de competición en karate: 1) kumite: combate entre dos competidores bajo una serie de reglas; y 2) kata: secuencia de técnicas y movimientos preestablecidos, ofensivos y defensivos ante un adversario imaginario (1).

En la modalidad de combate o kumite, se siguen unos criterios de puntuación que se dividen en: 1) Yuko (1 punto): cualquier golpe de puño directo o tsuki o indirecto o uchi, sobre cualquiera de las 7 zonas puntuables: cabeza, cara, cuello, abdomen, pecho, espalda y costados; 2) Waza-ari (2 puntos): patadas a nivel chudan: abdomen, pecho, espalda y costados; y 3) Ippon (3 puntos): patadas a nivel jodan (cara, cabeza y cuello) y cualquier técnica puntuable sobre un adversario que haya sido derribado, se haya caído o perdido el equilibrio (2). Además, el kumite puede realizarse de forma individual o por equipos. La categoría individual se divide en cadete (14-15 años), junior (16-17 años), sub21 (18-20 años) y sénior (+18 años), subdividiéndose cada categoría en distintos pesos (2).

Según la literatura consultada, durante un combate simulado se realizan alrededor de 11 a $21(16.3 \pm 5.1)$ acciones de alta intensidad, durando cada una de ellas de 1 a 3 segundos (3), mientras que en un combate internacional se llevan a cabo de 10 a $24(17 \pm 7)$ acciones con una duración de $<1$ a 5 segundos (4). La acción ofensiva y/o defensiva más corta, durante un combate simulado, dura $0.3 \pm 0.1$ segundos para combates de 2 y 3 minutos; y la más larga es de $2.1 \pm 1.0$ segundos y $1.8 \pm 0.4$ segundos, para los combates de 2 y 3 minutos respectivamente (5). 
Chaabène et al. (4) observaron que los karatekas emplean con mayor frecuencia las técnicas de miembros superiores. El puñetazo con el miembro superior adelantado a la cara denominado kisami-zuki fue la técnica más utilizada por los deportistas, la patada circular al tronco o mawashi-geri chudan fue la patada más usada y el puñetazo con el miembro superior adelantado seguido de puñetazo con el miembro superior atrasado a la cara o kisami kyaku-zuki jodan la combinación más utilizada. Sin embargo, no se encontraron diferencias estadísticamente significativas entre karatekas ganadores y perdedores en las respuestas fisiológicas, así como en las técnicas empleadas (4). Por otro lado, en relación con la puntuación, Riveiro et al. (6) observaron que el Yuko (puñetazo sobre cualquiera de las 7 zonas puntuables) es la forma de punto más frecuentada a nivel nacional e internacional, mientras que el Ippon (patadas a nivel de la cabeza o barridos) es más utilizado a nivel internacional frente al nacional. Además, los karatekas internacionales realizan más acciones puntuables que el grupo de karatekas de nivel nacional (6). Así pues, el karate es un deporte en el que se llevan a cabo acciones de alta intensidad, con desplazamientos explosivos de los miembros inferiores, y con predominio de las técnicas de puño.

Actualmente, la modalidad de kumite posee un reglamento específico desarrollado por la World Karate Federation (WKF) (2) para evitar contactos excesivos y agresiones entre deportistas. Arriaza et al. (7) estudiaron la incidencia de lesiones en karatekas, antes y después de la implementación de un nuevo reglamento de competición en el año 2000, obteniéndose una reducción de la tasa global de lesiones masculina y femenina de un $42 \%$ en comparación con la reglamentación anterior. Además, estás lesiones eran de carácter menor en su mayoría, siendo provocadas por puñetazos en el $67 \%$ de los casos (7). Según otros estudios, la tasa de lesiones ha sido de 20.2 lesiones por cada 100 atletas durante el entrenamiento (8); 9.87 la proporción de lesiones por cada 100 atletas y 45.26 la tasa de incidencia de lesiones por cada 1000 atletas en campeonatos nacionales de jóvenes karatekas (9); y 41.4 la incidencia de lesiones por cada 1000 atletas en campeonatos internacionales de alto nivel en jóvenes (16-20 años) (10).

La mayoría de estudios han encontrado que el mecanismo lesional más frecuente es la contusión (7-13), mientras que la zona corporal afectada en mayor número de ocasiones es la región de la cabeza, cara y cuello (7-10,1214). De todos estos estudios, sólo dos estudios, uno desarrollado en Irán y otro en Austria $(8,11)$, analizan las posibles lesiones durante los entrenamientos, afirmando que la mayoría de estas se producen durante el entrenamiento de combate o kumite.

El karate es pues un deporte con gran cantidad de federados con una reglamentación muy específica y una creciente proyección de futuro teniendo en cuenta su próxima participación en las olimpiadas. La modalidad de combate 0 kumite es la que mayor riesgo lesional implica (8). En este sentido, conocer la prevalencia de problemas como el dolor en esta población de deportistas durante el entrenamiento permitiría enfocar de una manera específica no solo su tratamiento fisioterapéutico sino su prevención, pudiendo así mejorar su rendimiento deportivo. 
El propósito de este estudio es conocer la prevalencia de dolor derivada del entrenamiento en karatekas que compiten en la modalidad de kumite o combate, así como la posible relación entre la presencia de dolor y sensación subjetiva de inestabilidad con las características antropométricas y deportivas.

\section{PARTICIPANTES, MATERIAL Y MÉTODOS}

\subsection{DISEÑO}

Se llevó a cabo un estudio de prevalencia entre enero y junio de 2018.

\subsection{PARTICIPANTES}

Se incluyeron todos aquellos sujetos, hombres y mujeres, pertenecientes a 7 clubes de karate adscritos a la federación madrileña de karate, competidores de la modalidad de combate (kumite), con un grado igual o superior a cinturón azul, dentro de las categorías cadete, junior, sub-21 y sénior (14 a >18 años).

Se excluyeron aquellos con cualquier fractura o intervención quirúrgica en el último año; práctica de otros deportes que superase en horas semanales al entrenamiento de karate; los menores de 14 años y los mayores de 50 años.

Los participantes que cumplieron ambos criterios de inclusión y exclusión, fueron informados acerca del propósito del estudio, dando su consentimiento informado por escrito para la participación en el mismo.

\subsection{RECOGIDA DE DATOS}

Se diseñó un cuestionario para la recogida de datos, teniendo en cuenta alguna de las variables analizadas en otros estudios similares acerca de la epidemiología de las lesiones derivadas del entrenamiento en karate (8) y de la prevalencia del dolor en otros deportes $(15,16)$. El cuestionario se entregó tras finalizar el entrenamiento en las instalaciones de cada club analizado, en presencia del investigador principal.

Las variables estudiadas incluían: 1) Factores antropométricos: edad, sexo, índice de masa corporal (IMC); 2) Datos deportivos: edad de comienzo en combate o kumite, grado de cinturón, competidor actual y práctica de otros deportes; 3) Entrenamientos: frecuencia y tiempo del entrenamiento de karate y pesas; estiramientos y tiempo de los mismos; 4) Dolor: presencia de dolor (actual, últimos 7 días o último mes), características (aparición en reposo, al realizar alguna técnica o durante alguna postura mantenida), intensidad subjetiva según la Escala Visual Analógica (EVA) (17) (durante el entrenamiento y en reposo), localización (mapa corporal) y limitación de los entrenamientos; 5) Sensación de inestabilidad en alguna articulación; y 6) Limitación de los entrenamientos por dolor durante la carrera deportiva (localización de los mismos en un mapa corporal). 


\subsection{ANÁLISIS DE DATOS}

Tanto las variables sociodemográficas como las variables resultado se han representado empleando la media aritmética y la desviación estándar (DE) o la mediana y el rango intercuartílico (IQR), dependiendo de la asunción o no, respectivamente, del supuesto de normalidad de las mismas determinado mediante el test de Kolmogorof-Smirnov (K-S). En el caso de las variables categóricas, se emplearon las frecuencias absolutas y relativas porcentuales.

Para determinar la medida de asociación entre dos variables categóricas, valorando el efecto mediante la diferencia de medias, se empleó la prueba de $X^{2}$ de Pearson o la prueba exacta de Fisher, cuando las variables eran dicotómicas. La asociación entre una variable dependiente dicotómica y dependiente cuantitativa de distribución paramétrica se llevó a cabo mediante el test t de Student para muestras independientes. Cuando la variable vulneraba el supuesto de normalidad (K-S), se emplearon la prueba U de Mann Whitney para dos muestras independientes y la prueba de Kruskal Wallis para tres muestras independientes, valorando la medida de efecto mediante la diferencia de medianas.

Como grado de significación estadística se empleó un valor de $p<0.05$ en todos los casos.

Se utilizó el programa Statistical Package for the Social Sciences software (SPSS $\circledast$ ) versión 24 para el análisis estadístico.

\section{RESULTADOS}

56 karatekas federados en la Comunidad de Madrid pertenecientes a las categorías cadete, junior, sub-21 y sénior fueron incluidos en el estudio. El flujo de participantes se puede observar en la Figura 1. Los sujetos considerados como pérdidas, rechazaron participar en el estudio $(n=3)$ o no acudieron al entrenamiento debido a causas ajenas al dolor $(n=4)$. Por otro lado, fueron excluidos aquellos participantes que fueron intervenidos quirúrgicamente en el último año $(n=2)$, los que practicaban otros deportes en mayor número de horas que el karate $(n=3)$ y aquellos con edades inferiores a 14 años $(n=4)$ o superiores a 50 años $(n=1)$. No se perdieron ninguno de los datos procedentes de las variables analizadas en los cuestionarios. 


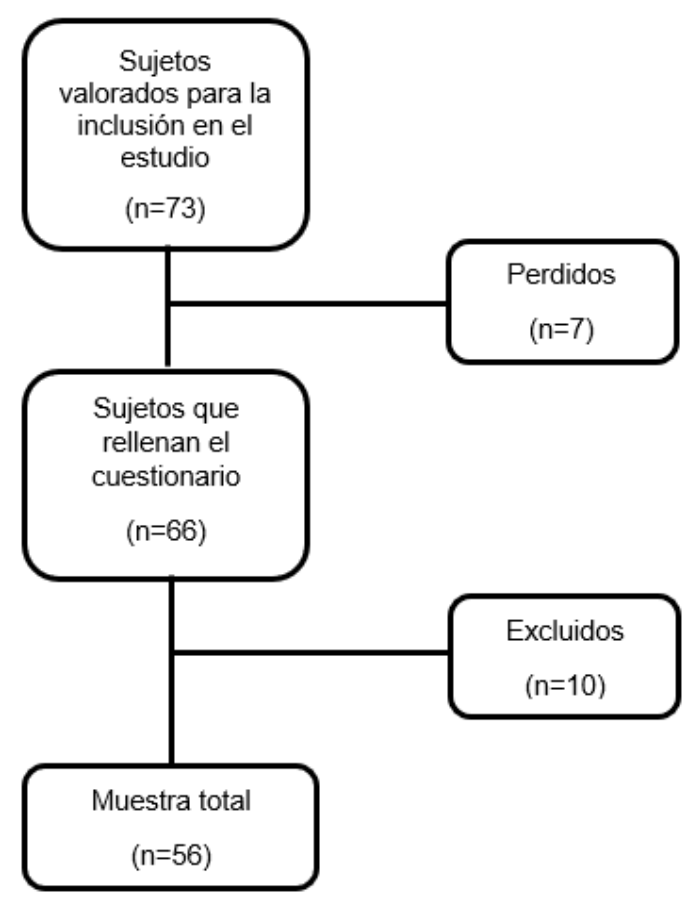

Figura 1. Flujo de participantes.

\subsection{DESCRIPCIÓN DE LA MUESTRA}

La muestra estuvo formada por 38 hombres $(67.9 \%)$ y 18 mujeres $(32.1 \%)$, con una media de edad de 22 (8) años. Los datos antropométricos se pueden observar en la Tabla 1.

Tabla 1. Características antropométricas de la muestra.

\begin{tabular}{|c|c|c|c|c|c|c|c|c|c|c|c|c|}
\hline \multirow[b]{2}{*}{$\begin{array}{l}\text { Media } \\
\text { (DE) }\end{array}$} & \multicolumn{3}{|c|}{ Muestra } & \multicolumn{3}{|c|}{ Cadete } & \multicolumn{3}{|c|}{ Junior } & \multicolumn{3}{|c|}{ Absoluto } \\
\hline & $\begin{array}{c}\text { Hombre } \\
(n=38)\end{array}$ & $\begin{array}{l}\text { Mujer } \\
(\mathrm{n}=18)\end{array}$ & $\begin{array}{l}\text { Total } \\
(n=56)\end{array}$ & $\begin{array}{c}\text { Hombre } \\
(n=5)\end{array}$ & $\begin{array}{c}\text { Mujer } \\
(n=4)\end{array}$ & $\begin{array}{l}\text { Total } \\
(n=9)\end{array}$ & $\begin{array}{c}\text { Hombre } \\
(n=7)\end{array}$ & $\begin{array}{l}\text { Mujer } \\
(n=5)\end{array}$ & $\begin{array}{l}\text { Total } \\
(\mathrm{n}=12) \\
\end{array}$ & $\begin{array}{c}\text { Hombre } \\
(\mathrm{n}=26)\end{array}$ & $\begin{array}{c}\text { Mujer } \\
(\mathrm{n}=9)\end{array}$ & $\begin{array}{l}\text { Total } \\
(\mathrm{n}=35)\end{array}$ \\
\hline $\begin{array}{l}\text { Edad } \\
\text { (años) }\end{array}$ & $23(9)$ & $18(3)$ & $22(8)$ & $14(0.5)$ & $\begin{array}{c}14 \\
(0.5) \\
\end{array}$ & $\begin{array}{c}14 \\
(0.5) \\
\end{array}$ & $16(0.4)$ & $\begin{array}{c}16 \\
(0.4) \\
\end{array}$ & $\begin{array}{c}16 \\
(0.4) \\
\end{array}$ & $27(9)$ & $21(2)$ & $26(8)$ \\
\hline $\begin{array}{l}\begin{array}{l}\text { Peso } \\
(\mathrm{kg})\end{array} \\
\end{array}$ & $\begin{array}{c}72.02 \\
(10.73) \\
\end{array}$ & $\begin{array}{l}57.97 \\
(7.24) \\
\end{array}$ & $\begin{array}{c}67.51 \\
(11.72) \\
\end{array}$ & $\begin{array}{l}62.2 \\
(9.8) \\
\end{array}$ & $\begin{array}{l}56.75 \\
(10.3) \\
\end{array}$ & $\begin{array}{c}59.77 \\
(9.8) \\
\end{array}$ & $\begin{array}{l}63.57 \\
(7.11) \\
\end{array}$ & $\begin{array}{l}59.26 \\
(8.74) \\
\end{array}$ & $\begin{array}{l}61.77 \\
(7.76) \\
\end{array}$ & $\begin{array}{l}76.19 \\
(9.25) \\
\end{array}$ & $\begin{array}{l}57.81 \\
(5.6) \\
\end{array}$ & $\begin{array}{c}71.46 \\
(11.69) \\
\end{array}$ \\
\hline $\begin{array}{l}\text { Altura } \\
\text { (cm) }\end{array}$ & $\begin{array}{c}174.92 \\
(6.87) \\
\end{array}$ & $\begin{array}{c}164 \\
(6.78)\end{array}$ & $\begin{array}{c}171.41 \\
(8.51) \\
\end{array}$ & $\begin{array}{l}170.2 \\
(8.07) \\
\end{array}$ & $\begin{array}{l}167.5 \\
(8.58) \\
\end{array}$ & $\begin{array}{c}169 \\
(7.89) \\
\end{array}$ & $\begin{array}{c}172.14 \\
(5.24) \\
\end{array}$ & $\begin{array}{c}163 \\
(5.09) \\
\end{array}$ & $\begin{array}{c}168.33 \\
(6.82)\end{array}$ & $\begin{array}{c}176.58 \\
(6.59) \\
\end{array}$ & $\begin{array}{c}163 \\
(7.01)\end{array}$ & $\begin{array}{c}173.09 \\
(8.93) \\
\end{array}$ \\
\hline $\begin{array}{l}\text { IMC } \\
\left(\mathrm{kg} / \mathrm{m}^{2}\right)\end{array}$ & $\begin{array}{l}23.45 \\
(2.58) \\
\end{array}$ & $\begin{array}{l}21.52 \\
(2.02) \\
\end{array}$ & $\begin{array}{l}22.83 \\
(2.56) \\
\end{array}$ & $\begin{array}{l}21.36 \\
(2.25) \\
\end{array}$ & $\begin{array}{l}20.07 \\
(1.64)\end{array}$ & $\begin{array}{c}20.78 \\
(2)\end{array}$ & $\begin{array}{l}21.42 \\
(1.91)\end{array}$ & $\begin{array}{l}22.31 \\
(3.12)\end{array}$ & $\begin{array}{l}21.79 \\
(2.39)\end{array}$ & $\begin{array}{l}24.4 \\
(2.29) \\
\end{array}$ & $\begin{array}{l}21.73 \\
(1.14) \\
\end{array}$ & $\begin{array}{l}23.71 \\
(2.36) \\
\end{array}$ \\
\hline
\end{tabular}

\subsection{DATOS DEPORTIVOS}

La muestra presentó una edad de comienzo en la modalidad de kumite de 13 (3) años, siendo el mínimo 6 años y el máximo 35 años de edad. El $48.6 \%$ de la muestra no practicaba otros deportes, mientras que de los que realizaban otros deportes, el $19.6 \%$ practicaba fútbol y el $17.9 \%$ atletismo. Los datos deportivos pueden observarse en la Tabla 2. Se han encontrado diferencias estadísticamente significativas entre el grado de cinturón y las categorías de combate $(p<0.01)$, es decir, cuanto mayor sea el grado de cinturón, mayor será la categoría de combate. 


\subsection{PREVALENCIA DE DOLOR Y DE INESTABILIDAD}

La prevalencia de dolor en la muestra ha sido del $67.9 \%(n=38)$. Se registraron 24 sujetos (42.9\%) que presentaron dolor en el momento del estudio. La categoría absoluta presentó mayor número de sujetos con dolor actual (70.8\%; $\mathrm{n}=17)$, seguido de la categoría junior con un $25 \%(\mathrm{n}=6)$ y por último la categoría cadete con un $4.2 \%(n=1)$. En cuanto a la variable dolor en los últimos 7 días 0 último mes, se registraron 38 sujetos $(67.9 \%)$, siendo mayoritaria la categoría absoluta con un $65.8 \%(n=25)$, seguido de la categoría junior con un $23.7 \%(n=9)$ y la categoría cadete con un $10.5 \%(n=4)$.

Un total de 7 sujetos (12.5\%) presentaron tres localizaciones de dolor diferentes, 9 deportistas (16.07\%) presentaron dos localizaciones de dolor, mientras que 22 karatekas (39.28\%) declararon una sola localización de dolor. En relación con la localización del dolor (Figura 2), la rodilla fue la zona más prevalente $(31 \%)$, seguida de la región del hombro (15\%), de la articulación metacarpofalángica del 1 을 dedo (11\%), y de la región del pie (11\%).

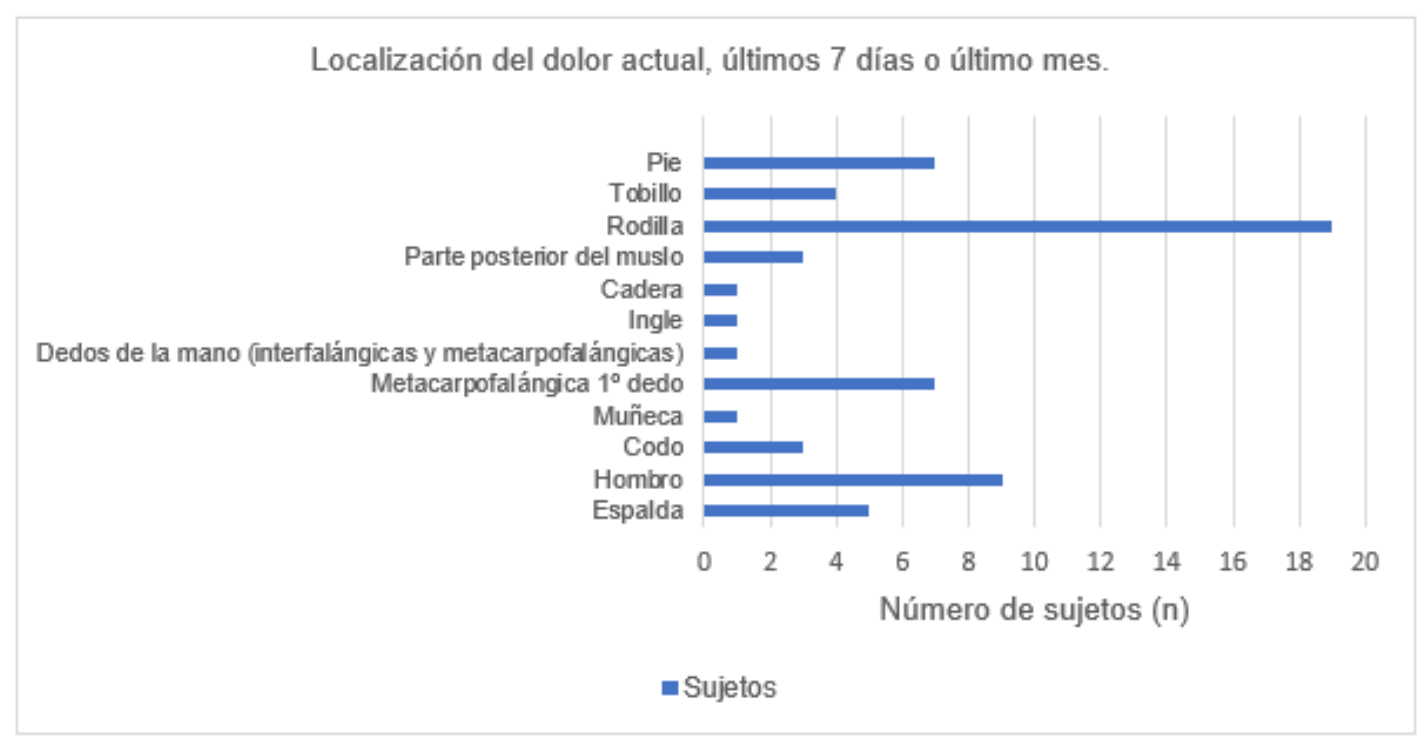

Figura 2. Localización del dolor actual, últimos 7 días o último mes.

Por otro lado, el dolor fue poco limitante en la mayoría de los participantes que refirieron dolor actual/ 7 días/mes, teniendo que interrumpir el entrenamiento temporalmente, y retomándolo en el $60.5 \%$ de los sujetos $(n=23)$. Únicamente en dos casos no pudieron continuar con el entrenamiento o no volvieron a entrenar por dolor (2.6\%). Además, el $80.4 \%(n=45)$ de los participantes afirmaron haber padecido dolores o molestias que les limitaron durante su carrera deportiva. El dolor más prevalente durante la carrera deportiva (Figura 3) fue la zona de la rodilla (32.1\%), seguida de la zona del tobillo $(28.6 \%)$ y de la de la espalda (25\%). Se registraron 28 sujetos $(50 \%)$ que presentaron sensación de inestabilidad en alguna articulación en el momento del estudio. La rodilla fue la articulación con mayor número de casos de inestabilidad con un $50 \%(n=14)$, seguida del hombro con un $17.9 \%(n=5)$ y del tobillo, con un $14.3 \%(n=4)$. 
Rev.int.med.cienc.act.fís.deporte - vol. 20 - número 78 - ISSN: 1577-0354

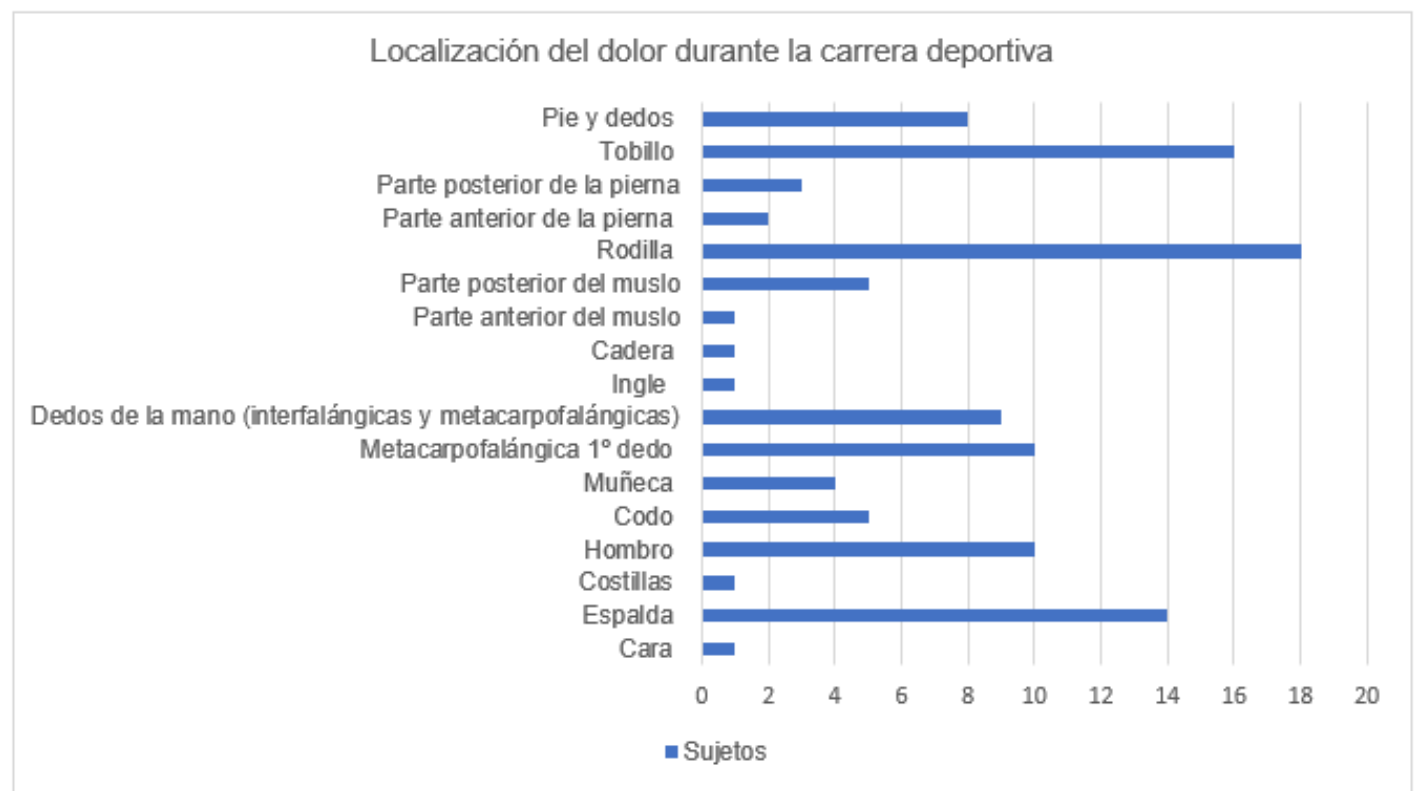

Figura 3. Localización del dolor durante la carrera deportiva. 
Rev.int.med.cienc.act.fís.deporte - vol. 20 - número 78 - ISSN: 1577-0354

Tabla 2. Características deportivas de la muestra.

\begin{tabular}{|c|c|c|c|c|c|c|}
\hline $\begin{array}{c}\text { Frecuencias absolutas } \\
\mathrm{n}(\%)\end{array}$ & & $\begin{array}{c}\text { Cadete } \\
(\mathrm{n}=9)\end{array}$ & $\begin{array}{l}\text { Junior } \\
(n=12)\end{array}$ & $\begin{array}{c}\text { Absoluto } \\
(\mathrm{n}=35)\end{array}$ & $\begin{array}{c}\text { Total } \\
(\mathrm{n}=56)\end{array}$ & $\mathrm{p}^{*}$ \\
\hline \multirow[t]{6}{*}{ Grado de cinturón } & Azul & $0(0)$ & $1(8.3)$ & $1(2.9)$ & $2(3.6)$ & $<0.01$ \\
\hline & Marrón & $4(44.4)$ & $1(8.3)$ & $2(5.7)$ & $7(12.5)$ & \\
\hline & Negro infantil & $5(55.6)$ & $5(41.7)$ & $0(0)$ & $10(17.9)$ & \\
\hline & Negro 1음 DAN & $0(0)$ & $5(41.7)$ & $15(42.9)$ & $20(35.7)$ & \\
\hline & Negro $2^{\circ}$ DAN & $0(0)$ & $0(0)$ & $9(25.7)$ & $9(16.1)$ & \\
\hline & $\begin{array}{l}\text { Negro } 3^{\circ} \text { DAN o } \\
\text { superior }\end{array}$ & $0(0)$ & $0(0)$ & $8(22.9)$ & $8(14.3)$ & \\
\hline \multirow[t]{2}{*}{ Competidor actual } & Sí & $8(88.9)$ & $10(83.3)$ & $31(88.6)$ & $49(87.5)$ & 0.886 \\
\hline & No & $1(11.1)$ & $2(16.7)$ & $4(11.4)$ & $7(12.5)$ & \\
\hline \multirow{3}{*}{$\begin{array}{l}\text { Frecuencia entrenamiento } \\
\text { karate }\end{array}$} & $1-2$ veces & $1(11.1)$ & $1(8.3)$ & $2(5.7)$ & $4(7.1)$ & 0.613 \\
\hline & 3-4 veces & $7(77.8)$ & $8(66.7)$ & $30(85.7)$ & $45(80.4)$ & \\
\hline & 5 o más veces & $1(11.1)$ & $3(25)$ & $3(8.6)$ & $7(12.5)$ & \\
\hline \multirow{2}{*}{$\begin{array}{c}\text { Entrenamiento más de una } \\
\text { vez al día }\end{array}$} & Sí & $0(0)$ & $3(25)$ & $6(17.1)$ & $9(16.1)$ & 0.292 \\
\hline & No & $9(100)$ & $9(75)$ & $29(82.9)$ & 47 (83.9) & \\
\hline \multirow[t]{3}{*}{ Tiempo entrenamiento karate } & $30-60$ minutos & $1(11.1)$ & $3(25)$ & $7(20)$ & $11(19.6)$ & 0.398 \\
\hline & $60-90$ minutos & $5(55.6)$ & $7(58.3)$ & $25(71.4)$ & $37(66.1)$ & \\
\hline & Más de 90 minutos & $3(33.3)$ & $2(16.7)$ & $3(8.6)$ & $8(14.3)$ & \\
\hline \multirow[t]{3}{*}{ Entrenamiento pesas } & No & $6(66.7)$ & $11(91.7)$ & $21(60)$ & $38(67.9)$ & 0.368 \\
\hline & $1-2$ veces & $2(22.2)$ & $1(8.3)$ & $9(25.7)$ & $12(21.4)$ & \\
\hline & 3-4 veces & $1(11.1)$ & $0(0)$ & $5(14.3)$ & $6(10.7)$ & \\
\hline \multirow[t]{3}{*}{ Tiempo entrenamiento pesas } & 30 minutos & $2(66.7)$ & $0(0)$ & $4(28.6)$ & $6(33.3)$ & 0.423 \\
\hline & $30-60$ minutos & $0(0)$ & $0(0)$ & $5(35.7)$ & $5(27.8)$ & \\
\hline & $60-90$ minutos & $1(33.3)$ & $1(100)$ & $5(35.7)$ & $7(38.9)$ & \\
\hline \multirow[t]{3}{*}{ Estiramientos } & No & $0(0)$ & $1(8.3)$ & $6(17.1)$ & $7(12.5)$ & 0.334 \\
\hline & $\begin{array}{l}\text { Sí, solamente de las } \\
\text { piernas }\end{array}$ & $2(22.2)$ & $6(50)$ & $11(31.4)$ & 19 (33.9) & \\
\hline & $\begin{array}{c}\text { Sí, de los brazos y las } \\
\text { piernas }\end{array}$ & $7(77.8)$ & $5(41.7)$ & $18(51.4)$ & $30(53.6)$ & \\
\hline \multirow[t]{4}{*}{ Tiempo estiramientos } & 5 minutos & $4(44.4)$ & $6(54.5)$ & $9(31)$ & $19(38.8)$ & 0.718 \\
\hline & 10 minutos & $5(55.6)$ & $4(36.4)$ & $15(51.7)$ & $24(49)$ & \\
\hline & 15 minutos & $0(0)$ & $1(9.1)$ & $4(13.8)$ & $5(10.2)$ & \\
\hline & 20 minutos o más & $0(0)$ & $0(0)$ & $1(3.4)$ & $1(2)$ & \\
\hline
\end{tabular}




\subsection{CARACTERÍSTICAS DEL DOLOR}

Se han encontrado 18 sujetos (32.1\%) que presentaban dolor en reposo y 32 sujetos $(57.1 \%)$ con dolor durante la realización de alguna técnica en el momento del estudio. La técnica con mayor número de casos con dolor fue kisami-zuki o puñetazo con el miembro superior adelantado (46.9\%), seguido de gyaku-zuki o puñetazo con el miembro superior atrasado (40.6\%), y mawashi-geri o patada circular con el empeine (40.6\%). Por otro lado, en cuanto a las posturas dolorosas, seiza (postura durante el saludo inicial) fue la seleccionada en mayor número de ocasiones por 8 sujetos (14.3\%).

El dolor en la muestra obtuvo una intensidad durante el entrenamiento de 4.6 (2.8) $\mathrm{cm}$ (mediana y rango intercuartílico), mientras que en reposo fue de 0.8 (2.7) $\mathrm{cm}$. La intensidad del dolor en función de la categoría y del sexo puede observarse en la Tabla 3 . No se han encontrado diferencias estadísticamente significativas ni entre el dolor y las categorías, ni entre el dolor y el sexo. Aunque no se han hallado diferencias, las mujeres y los karatekas junior presentan una mayor intensidad de dolor durante el entrenamiento.

Tabla 3. Intensidad del dolor según categoría y sexo.

\begin{tabular}{|c|c|c|c|c|c|c|c|}
\hline \multirow{2}{*}{ Mediana (IQR) } & \multicolumn{3}{|c|}{ Categoría } & \multirow[t]{2}{*}{$p$} & \multicolumn{2}{|c|}{ Sexo } & \multirow[t]{2}{*}{$p$} \\
\hline & Cadete & Junior & Absoluto & & Hombre & Mujer & \\
\hline $\begin{array}{c}\text { EVA } \\
\text { Entrenamiento, } \\
\mathrm{cm} .\end{array}$ & $4.7(1.8)$ & $5(2.7)$ & $4.1(3.2)$ & $0.753^{a}$ & $4.1(3.4)$ & $4.8(2.8)$ & $0.083^{b}$ \\
\hline $\begin{array}{c}\text { EVA } \\
\text { En este momento, } \\
\mathrm{cm} .\end{array}$ & 2.7 (3.2) & $0.7(1.5)$ & $0.9(2.6)$ & $0.851^{a}$ & $0.7(2.5)$ & $1.1(5.1)$ & $0.243^{b}$ \\
\hline
\end{tabular}

\subsection{DOLOR E INESTABILIDAD CON RELACIÓN A FACTORES ANTROPOMÉTRICOS Y DEPORTIVOS}

Con respecto a la variable dolor actual y los factores antropométricos (Tabla 4), se han encontrado diferencias estadísticamente significativas en función de la edad ( $p=0.04)$ y del IMC $(p=0.045)$ de la muestra estudiada, es decir, a mayor edad y a mayor IMC, más casos de dolor. En cuanto al dolor en los últimos 7 días o último mes y a la inestabilidad frente a los factores antropométricos, las diferencias encontradas no fueron estadísticamente significativas (Tabla 4).

Por otro lado, en relación con los datos deportivos (Tablas 4 y 5), el dolor actual mostró diferencias estadísticamente significativas con respecto al grado de cinturón, es decir, a mayor grado de cinturón, más casos de dolor $(p=0.017)$. Además, se encontraron diferencias estadísticamente significativas entre el dolor últimos 7 días o último mes y la frecuencia de entrenamiento de karate $(p=0.011)$, de modo que, a mayor frecuencia de entrenamiento, más casos de dolor. Para el resto de variables, no se encontraron diferencias. 
Rev.int.med.cienc.act.fís.deporte - vol. 20 - número 78 - ISSN: 1577-0354

Tabla 4. Dolor e inestabilidad con relación a factores antropométricos y deportivos.

\begin{tabular}{|c|c|c|c|c|c|c|c|c|c|}
\hline & \multicolumn{3}{|c|}{ Dolor actual } & \multicolumn{3}{|c|}{ Dolor 7 días o mes } & \multicolumn{3}{|c|}{ Inestabilidad } \\
\hline & Sí $(n=24)$ & No $(n=32)$ & $p$ & Sí (n=38) & No $(n=18)$ & $p$ & Sí (n=28) & No $(n=28)$ & $p$ \\
\hline \multicolumn{10}{|l|}{$\begin{array}{l}\text { Factores } \\
\text { antropométricos. Media } \\
\text { (DE) }\end{array}$} \\
\hline Edad, años. & $24(9)$ & $20(7)$ & $0.04^{a}$ & $23(8)$ & $20(8)$ & $0.261^{a}$ & $23(8)$ & $21(8)$ & $0.348^{a}$ \\
\hline Peso, kg. & $\begin{array}{l}70.76 \\
(13.23)\end{array}$ & $65.06(9.98)$ & $0.072^{\mathrm{a}}$ & $\begin{array}{c}68.84 \\
(12.11)\end{array}$ & $\begin{array}{c}64.69 \\
(10.64)\end{array}$ & $0.219^{a}$ & $70.04(11.4)$ & $\begin{array}{c}64.97 \\
(11.69)\end{array}$ & $0.106^{\mathrm{a}}$ \\
\hline Altura, $\mathrm{cm}$. & $\begin{array}{c}172.33 \\
(8.87)\end{array}$ & $\begin{array}{c}170.72 \\
(8.13)\end{array}$ & $0.488^{a}$ & $\begin{array}{l}172.34 \\
(8.46)\end{array}$ & $\begin{array}{l}169.44 \\
(8.53)\end{array}$ & $0.238^{a}$ & $172.5(8.23)$ & $\begin{array}{c}170.32 \\
(8.8)\end{array}$ & $0.343^{a}$ \\
\hline IMC, $\mathrm{kg} / \mathrm{m}^{2}$. & $23.67(3)$ & $22.2(2.01)$ & $0.045^{a}$ & $23.04(2.73)$ & $\begin{array}{l}22.38 \\
(2.19)\end{array}$ & $0.370^{\mathrm{a}}$ & $23.43(2.64)$ & $\begin{array}{l}22.23 \\
(2.38)\end{array}$ & $0.08^{a}$ \\
\hline $\begin{array}{l}\text { Sexo (hombre/mujer). } \\
\text { Frecuencia absoluta. }\end{array}$ & $18 / 6$ & $20 / 12$ & $0.322^{b}$ & $27 / 11$ & $11 / 7$ & $0.457^{b}$ & $20 / 8$ & $18 / 10$ & $0.567^{b}$ \\
\hline \multicolumn{10}{|l|}{$\begin{array}{l}\text { Datos deportivos. } \\
\text { Frecuencias absolutas. }\end{array}$} \\
\hline $\begin{array}{l}\text { Categoría } \\
\text { (cadete/junior/absoluto). }\end{array}$ & $1 / 6 / 17$ & $8 / 6 / 18$ & $0.11^{b}$ & $4 / 9 / 25$ & $5 / 3 / 10$ & $0.253^{\mathrm{b}}$ & $2 / 8 / 18$ & $7 / 4 / 17$ & $0.126^{\mathrm{b}}$ \\
\hline $\begin{array}{l}\text { Grado } \\
\text { (azul/marrón/negro } \\
\text { infantil/negro } 1^{\circ}{ }^{\circ} \\
\text { DAN/negro } 2^{\circ} \\
\text { DAN/negro } 3^{\circ} \text { DAN o } \\
\text { superior }\end{array}$ & $1 / 0 / 4 / 10 / 2 / 7$ & $1 / 7 / 6 / 10 / 7 / 1$ & $0.017^{b}$ & $1 / 3 / 7 / 15 / 4 / 8$ & $1 / 4 / 3 / 5 / 5 / 0$ & $0.116^{b}$ & $0 / 1 / 5 / 13 / 3 / 6$ & $2 / 6 / 5 / 7 / 6 / 2$ & $0.065^{b}$ \\
\hline Competidor actual (sí/no) & $20 / 4$ & $29 / 3$ & $0.414^{b}$ & $34 / 4$ & $15 / 3$ & $0.516^{b}$ & $24 / 4$ & $25 / 3$ & $0.686^{b}$ \\
\hline $\begin{array}{l}\text { Frecuencia } \\
\text { entrenamiento karate (1- } \\
2 \text { veces } / 3-4 \text { veces } / 5 \text { o } \\
\text { más veces) }\end{array}$ & $0 / 21 / 3$ & $4 / 24 / 4$ & $0.195^{b}$ & $0 / 33 / 5$ & $4 / 12 / 2$ & $0.011^{b}$ & $1 / 26 / 1$ & $3 / 19 / 6$ & $0.059^{b}$ \\
\hline $\begin{array}{l}\text { Tiempo entrenamiento } \\
\text { karate }(30-60 \\
\text { minutos } / 60-90 \\
\text { minutos/más de } 90 \\
\text { minutos). }\end{array}$ & $6 / 16 / 2$ & $5 / 21 / 6$ & $0.437^{b}$ & $7 / 26 / 5$ & $4 / 11 / 3$ & $0.863^{b}$ & $6 / 18 / 4$ & $5 / 19 / 4$ & $0.943^{b}$ \\
\hline
\end{tabular}


Rev.int.med.cienc.act.fís.deporte - vol. 20 - número 78 - ISSN: 1577-0354

Tabla 5 (continuación). Dolor e inestabilidad con relación a factores antropométricos y deportivos.

\begin{tabular}{|c|c|c|c|c|c|c|c|c|c|}
\hline $\begin{array}{l}\text { Entrenamiento pesas } \\
\text { (no/1-2 veces/3-4 } \\
\text { veces). }\end{array}$ & $13 / 8 / 3$ & $25 / 4 / 3$ & $0.131^{b}$ & $23 / 10 / 5$ & $15 / 2 / 1$ & $0.233^{b}$ & $18 / 6 / 4$ & $20 / 6 / 2$ & $0.68^{b}$ \\
\hline $\begin{array}{l}\text { Estiramientos (no/sí, } \\
\text { solamente de las } \\
\text { piernas/sí, de los brazos } \\
\text { y las piernas). }\end{array}$ & $3 / 10 / 11$ & $4 / 9 / 19$ & $0.546^{b}$ & $6 / 13 / 19$ & $1 / 6 / 11$ & $0.520^{\mathrm{b}}$ & $2 / 11 / 15$ & $5 / 8 / 15$ & $0.415^{b}$ \\
\hline $\begin{array}{l}\text { Tiempo estiramientos (5 } \\
\text { minutos } / 10 \text { minutos } / 15 \\
\text { minutos/20 minutos o } \\
\text { más) }\end{array}$ & $7 / 11 / 2 / 1$ & $12 / 13 / 3 / 0$ & $0.633^{b}$ & $12 / 15 / 4 / 1$ & $7 / 9 / 1 / 0$ & $0.770^{\mathrm{b}}$ & $12 / 12 / 2 / 0$ & $7 / 12 / 3 / 1$ & $0.505^{b}$ \\
\hline $\begin{array}{l}\text { Limitación por dolor } \\
\text { (no/sí, he tenido que } \\
\text { parar temporalmente, } \\
\text { pero he podido retomar } \\
\text { el entrenamiento/sí, no } \\
\text { pude continuar el } \\
\text { entrenamiento/sí, no he } \\
\text { vuelto a entrenar por mi } \\
\text { dolor). }\end{array}$ & $7 / 16 / 0 / 1$ & $17 / 14 / 1 / 0$ & $0.153^{b}$ & $13 / 23 / 1 / 1$ & $11 / 7 / 0 / 0$ & $0.253^{b}$ & $9 / 18 / 0 / 1$ & $15 / 12 / 1 / 0$ & $0.195^{b}$ \\
\hline
\end{tabular}

a Test t de Student, ${ }^{b}$ Prueba de $x^{2}$ de Pearson, ${ }^{c}$ Prueba exacta de Fisher 


\subsection{TAMAÑO MUESTRAL PARA UN FUTURO ESTUDIO DE PREVALENCIA}

Asumiendo que la población es infinita, se precisa de una muestra aleatoria de 335 sujetos para estimar un porcentaje poblacional del $67.9 \%$, con una confianza del $95 \%$ y una precisión de +/- 5 unidades porcentuales con una tasa de reposición de $0 \%$.

\section{DISCUSIÓN}

Según el conocimiento de los autores, se trata del primer estudio de prevalencia de dolor durante el entrenamiento en karatekas de combate desarrollado en España. Por otro lado, aunque se han encontrado otros estudios sobre karatekas de competición o kumite, se trata de estudios epidemiológicos que recogen las lesiones principalmente en competición $(7,9-11,18)$. En cuanto a los dos únicos estudios hallados sobre prevalencia de lesiones en los entrenamientos $(8,11)$ estos presentan los porcentajes de lesiones de manera genérica en base a sus mecanismos lesionales, así como su localización de forma inespecífica, sin recabar el dolor u otras sensaciones subjetivas como la inestabilidad en zonas anatómicas específicas.

La prevalencia del dolor en este estudio se ha dividido en tres variables: presencia de dolor, dolor actual y dolor en los últimos 7 días o último mes. La presencia de dolor se ha basado en cualquier caso positivo presente tanto en la variable dolor actual, como en dolor en los últimos 7 días o último mes, obteniéndose una prevalencia del $67.9 \%$. Los resultados obtenidos muestran más prevalente a la categoría absoluta.

A pesar de no obtenerse diferencias estadísticamente significativas entre sexos tanto en la presencia de dolor como en su frecuencia según categorías, la media de edad de los hombres fue 5 años superior a la de las mujeres que forman el estudio. Estos resultados son similares a los de otros estudios $(7,13)$ que muestran una tasa de lesiones similares en hombres y mujeres. También contrasta con estudios $(10,12)$ cuyos resultados muestran una tasa inferior en mujeres, o incluso un mayor riesgo de lesiones en mujeres de entre 12-17 años (9). Las diferencias con estos últimos pueden deberse al muestreo empleado en el presente estudio, ya que, al tratarse de un muestreo consecutivo no probabilístico, los participantes son en su mayoría hombres y mayores de 18 años. Además, la diferencia de edad entre hombres y mujeres de la muestra podría dar lugar a una experiencia distinta en competición.

En el caso de los hombres del presente estudio, como presentan una mayor edad, tienen mayor experiencia en combate. Los karatekas con menor experiencia deportiva han mostrado tener una mayor tasa de lesiones (8), puesto que, a mayor grado de cinturón, menor tiempo de reacción (19-21) y mayor velocidad de percepción visual (22) teniendo así mayor posibilidad de evitar los contactos del adversario, y por lo tanto menor riesgo de lesión. Sin embargo, contrastando con lo expuesto, otro estudio (10) muestra mayor tasa de lesiones 
en deportistas con mayor experiencia, ya que potencialmente pueden desarrollar técnicas ofensivas peligrosas y defensivas con mayor fuerza y rapidez.

En el presente estudio, el dolor ha resultado poco limitante en el $60.5 \%$ de la muestra. Esto coincide con la mayoría de estudios (7-13), donde las lesiones son de carácter menor, siendo mayoritariamente contusiones. Asimismo, puesto que la intensidad subjetiva de dolor ha resultado ser moderada (23), esto podría explicar también el hecho de que hayan resultado ser poco limitantes, junto con el hecho de que los deportistas que realizan deportes de contacto se vuelven más tolerantes al dolor que los no realizan este tipo de deportes (24).

En cuanto a la localización, la región de la rodilla ha resultado ser la zona de dolor principal más prevalente en el momento del estudio $(36.8 \%)$ y durante la carrera deportiva (32.1\%). Esto coincide con los resultados de Tischer et al. (18), que afirma que la rodilla fue la región con mayor número de atenciones médicas en el Campeonato del Mundo de karate de 2014, tanto en kata como en kumite (28.7\% y $26.7 \%$ respectivamente). Sin embargo, también contrasta con la mayoría de estudios (7-10,12-14) realizados en competiciones y durante el entrenamiento, que han registrado la región de la cabeza como la zona más comúnmente lesionada. Esto puede deberse a que, en competición, la técnica más empleada es el kisami-zuki (4) o puñetazo con el miembro superior adelantado, mientras que el Yuko o puñetazo a cualquiera de las 7 zonas puntuables, es el punto más usado (6), realizando esta técnica a la cara del adversario, y, por lo tanto, generando una mayor exposición a contusiones en esta zona. Aunque esta técnica dirigida a la cara es la más utilizada en competición, durante el entrenamiento, se suelen evitar los contactos excesivos a la cara de los compañeros de club.

Además, en la rodilla, el sobreuso o la excesiva solicitación de esta articulación podría ser una de las causas del origen de dolor, debido a que se llevan a cabo desplazamientos rápidos en competición o kumite (25) y técnicas (6) que solicitan la citada articulación, además de necesitar un control excéntrico para frenar las patadas (26), evitando así los contactos excesivos. En taekwondo se llevó a cabo un estudio (27) donde se observó una alta prevalencia de dolor crónico por sobreuso (65.8\%), principalmente en el tren inferior (61.5\%) y en las rodillas $(26.4 \%)$. El taekwondo podría asimilarse al karate por realizarse técnicas de patada parecidas, además de llevar a cabo unos desplazamientos y un bote durante la guardia en reposo similar.

Por otro lado, en relación con la inestabilidad, el $50 \%$ de los sujetos presentaron esta sensación, siendo la rodilla la articulación con más casos de inestabilidad (50\%). Según el estudio de Solis et al. (28), cuanto mayor IMC y más edad, mayor disfunción en la rodilla. Esto coincide con los resultados del presente estudio, donde los sujetos con inestabilidad presentaban mayor IMC y mayor edad, aunque no se han obtenido diferencias estadísticamente significativas. Pop et al. (29) encontraron que el grupo de karate comprendido entre 9-19 años, presentaba mejor estabilidad corporal frente a un grupo control de entre 10-16 años que no realizaba actividad física. Esto podría sugerir, que los karatekas, en este caso menores de 19 años, presentan una mayor estabilidad de rodilla frente a la población sedentaria. 
En cuanto al momento de aparición de dolor, la mayor parte de sujetos con dolor lo han manifestado durante la realización de técnicas de karate (57.1\%) frente al dolor en reposo (32.1\%). La técnica más dolorosa ha resultado ser kisami-zuki o puñetazo con el miembro superior adelantado (46.9\%), seguido de gyaku-zuki o puñetazo con el miembro superior atrasado y mawashi gerio patada circular con el empeine (40.6\% ambos). Estas técnicas coinciden con el estudio de Chaabène et al. (4) en ser las más utilizadas en kumite. Además, los karatekas en kumite llevan a cabo técnicas de miembros superiores en mayor medida que de miembros inferiores (4), siendo el Yuko la forma de puntuar más utilizada a nivel nacional e internacional (6). El sobreuso de estas técnicas podría explicar el dolor durante la realización de las mismas, tal y como parece indicar el estudio de Koh sobre prevalencia del dolor por sobreuso en taekwondo (27).

En cuanto a la relación entre el dolor e inestabilidad con los factores antropométricos, se han encontrado diferencias estadísticamente significativas en la variable dolor actual, de forma que a mayor edad y mayor IMC más casos de dolor. En cuanto a la edad, la media de los sujetos con dolor se asemeja a lo observado en un estudio retrospectivo (12) donde se mostraba una alta tasa de lesión en torno a los 20-24 años. Langley et al. (30) afirman que la asociación entre prevalencia del dolor en España y edad no está del todo clara, sin embargo, en su estudio resultaron padecer dolor con mayor frecuencia la población comprendida entre 40 y 59 años, resultando una edad superior a la media de la muestra del presente estudio.

Respecto al IMC, se observa que, en la muestra, los sujetos con dolor tienen de media $5 \mathrm{~kg}$ más que los sujetos sin dolor (70vs65kg). Esto difiere del estudio de Ziaee et al. durante el entrenamiento de karate, donde el rango de peso con mayor número de lesiones se situaba entre 50 y $60 \mathrm{~kg}$ (8). Las diferencias con el presente estudio podrían deberse a la edad de la muestra, ya que el $46 \%$ de la muestra del anterior estudio (8) se situaba entre 14 y 19 años. Asimismo, la diferencia en relación con la experiencia (menor de 3 años en el $88 \%$ de los deportistas lesionados (8) frente a los 13 años de edad media de comienzo en kumite del presente estudio) podría explicar el mayor riesgo lesional.

En competición, en la categoría sub-21, se registró una mayor tasa de lesiones cuando la competición estaba dividida en tres categorías de pesos, en lugar de cinco a partir del año 2015, pudiendo deberse al enfrentamiento de deportistas con mayor diferencia entre pesos dentro de la misma categoría (10). Además, el estudio de Solis et al. (28), expone que cuanto mayor es el IMC, mayor sintomatología y disfuncionalidad de la rodilla. Esto coincide con los resultados del presente estudio, dado que tanto la zona de dolor como la zona de inestabilidad más prevalente ha resultado ser la región de rodilla, y que las personas con inestabilidad y dolor, presentan unos valores de IMC superiores. La articulación de la rodilla soporta una carga mayor en estos sujetos, pudiendo generar así mayor riesgo de dolor e inestabilidad.

Con respecto a los datos deportivos, se han encontrado diferencias estadísticamente significativas entre el dolor actual y el grado de cinturón. Cuanto mayor grado de cinturón, mayor número de casos de dolor. Esto puede 
deberse a que, en la categoría absoluta, donde predominan grados altos, el tiempo de combate aumenta a 3 minutos (2) y aumenta el tiempo de exposición del deportista, aumentando así su riesgo lesional. Sin embargo, hay que tener en cuenta que la muestra no está balanceada en función del grado de cinturón, ya que según la edad de los participantes del estudio no pueden optar a cualquier cinturón.

También se han encontrado diferencias estadísticamente significativas entre el dolor en los últimos 7 días o último mes y la frecuencia de entrenamiento de karate de modo que, a mayor frecuencia de entrenamiento, mayor dolor. La mayor parte de la población del estudio tiende a entrenar 3-4 veces por semana. Al entrenar un mayor número de horas, aumenta el tiempo de exposición a lesiones durante la práctica de kumite. También habría que tener en cuenta, que los sujetos del estudio son competidores actuales en su mayoría (49vs7), lo que podría influir en un mayor número de horas de exposición a lesiones durante las competiciones y durante el entrenamiento.

Este estudio no está exento de limitaciones, la principal limitación se basa en su diseño, ya que, al tratarse de un estudio transversal, las asociaciones entre el dolor y las variables estudiadas no permiten establecer relaciones causales. Serían necesarios más estudios con un mayor tamaño muestral de forma balanceada, en cuanto a edad, sexo y categorías de competición, con un diseño longitudinal, para confirmar los resultados del presente estudio y extrapolarlos a la población de karatekas.

\section{CONCLUSIONES}

El dolor en karatekas de competición durante los entrenamientos es un problema frecuente que muestra una alta prevalencia en la muestra estudiada. La rodilla resulta ser la zona más afectada tanto por dolor como por inestabilidad. El dolor se relaciona significativamente con aquellos karatekas con un mayor índice de masa corporal, mayor edad, mayor grado de cinturón y mayor frecuencia de entrenamiento. Además, el dolor parece ser moderado y se presenta principalmente con la técnica de puñetazo con el miembro superior adelantado o kisami-zuki.

\section{REFERENCIAS BIBLIOGRÁFICAS}

(1) Chaabene H, Hachana Y, Franchini E, Mkaouer B, Chamari K. Physical and physiological profile of elite karate athletes. Sports Med 2012; 42 (10): 829843. https://doi.org/10.1007/BF03262297

(2) World Karate Federation. Reglas de competición de kumite y kata [Internet]. [consultado 17 junio 2018]. Disponible en: https://www.wkf.net/pdf/WKFCompetitionRules2018 ESP.pdf

(3) Beneke R, Beyer T, Jachner C, Erasmus J, Hütler M. Energetics of karate kumite. Eur J Appl Physiol 2004; 92 (4-5): 518-523. https://doi.org/10.1007/s00421004-1073-x

(4) Chaabène H, Franchini $E$, Miarka $B$, Selmi MA, Mkaouer B, Chamari K. Timemotion analysis and physiological responses to karate official combat sessions: is there a difference between winners and defeated karatekas? Int 
J Sports Physiol Perform 2014; 9 (2): 302-308. https://doi.org/10.1123/ijspp.2012-0353

(5) lide K, Imamura H, Yoshimura Y, Yamashita A, Miyahara K, Miyamoto N, et al. Physiological responses of simulated karate sparring matches in young men and boys. J Strength Cond Res 2008; 22 (3): 839-844. https://doi.org/10.1519/JSC.0b013e31816a5af6

(6) Riveiro-Bozada A, García-García O, Serrano-Gómez V, Morales-Sánchez V, López-Lopez JA, Hernández-Mendo A. Influencia del nivel de competición en las acciones técnicas de punto realizadas en Shiai Kumite femenino de karate: análisis de coordenadas polares. CPD 2016; 16 (1): 51-68.

(7) Arriaza R, Leyes M, Zaeimkohan H, Arriaza A. The injury profile of Karate World Championships: new rules, less injuries. Knee Surg Sports Traumatol Arthrosc 2009; 17 (12): 1437-1442. https://doi.org/10.1007/s00167-0090856-3

(8) Ziaee V, Shobbar M, Lotfian S, Ahmadinejad M. Sport Injuries of Karate During Training: An Epidemiologic Study in Iran. Asian J Sports Med 2015; 6 (2): e26832. https://doi.org/10.5812/asjsm.26832

(9) Čierna D, Lystad RP. Epidemiology of competition injuries in youth karate athletes: a prospective cohort study. Br J Sports Med 2017; 51 (17): 12851288. https://doi.org/10.1136/bjsports-2017-097603

(10) Čierna D, Barrientos M, Agrasar C, Arriaza R. Epidemiology of injuries in juniors participating in top-level karate competition: a prospective cohort study. $\mathrm{Br} \mathrm{J}$ Sports Med 2018; 52 (11): 730-734. https://doi.org/10.1136/bjsports-2017$\underline{097756}$

(11) Greier K, Riechelmann H, Ziemska J. Sport injuries in full contact and semicontact karate. Sportverletz Sportschaden 2014; 28 (1): 31-35. https://doi.org/10.1055/s-0033-1356192

(12) Sterkowicz S, Sterkowicz-Przybycień K. Injuries in karate: A review. OA Sports Medicine 2013; 1 (2): 14. https://doi.org/10.13172/2053-2040-1-2-771

(13) Thomas RE, Ornstein J. Injuries in karate: systematic review. Phys Sportsmed 2018; 46 (3): 279-303. https://doi.org/10.1080/00913847.2018.1472510

(14) Vidovic-Stesevic V, Verna C, Krastl G, Kuhl S, Filippi A. Facial and Dental Injuries Facial and Dental Injuries in Karate. Swiss Dent J 2015; 125 (7-8): 810-814.

(15) Bailón-Cerezo J, Torres-Lacomba M, Gutiérrez-Ortega C. Prevalencia del dolor de hombro en nadadores de competición: estudio piloto / Shoulder Pain Prevalence in Competitive Swimmers: A Pilot Study. Revista Internacional de Medicina y Ciencias de la Actividad Física y del Deporte 2016; 16 (62): 317334. https://doi.org/10.15366/rimcafd2016.62.009

(16) Pérez Costa E, Torres Lacomba M, Gutiérrez Ortega C. Prevalencia de dolor de tobillo en futbolistas de competición: estudio piloto transversal. Fisioterapia 2017; 39 (1): 25-32. https://doi.org/10.1016//.ft.2016.01.001

(17) Alghadir AH, Anwer S, lqbal A, lqbal ZA. Test-retest reliability, validity, and minimum detectable change of visual analog, numerical rating, and verbal rating scales for measurement of osteoarthritic knee pain. J Pain Res 2018; 11: 851-856. https://doi.org/10.2147/JPR.S158847

(18) Tischer T, Lembcke B, Ellenrieder M, Glass Ä, Weigert W, Mittelmeier W. [Injuries in Karate Sports: A Survey Performed During the World Championship 2014]. Sportverletz Sportschaden 2016; 30 (4): 204-210. https://doi.org/10.1055/s-0042-112689 
(19) Fontani G, Lodi L, Felici A, Migliorini S, Corradeschi F. Attention in athletes of high and low experience engaged in different open skill sports. Percept Mot Skills 2006; 102 (3): 791-805. https://doi.org/10.2466/pms.102.3.791-805

(20) Mori S, Ohtani Y, Imanaka K. Reaction times and anticipatory skills of karate athletes. Hum Mov Sci 2002; 21 (2): 213-230. https://doi.org/10.1016/S01679457(02)00103-3

(21) Moscatelli F, Messina G, Valenzano A, Petito A, Triggiani Al, Messina A, et al. Differences in corticospinal system activity and reaction response between karate athletes and non-athletes. Neurol Sci 2016; 37 (12): 1947-1953. https://doi.org/10.1007/s10072-016-2693-8

(22) Kim HS, Petrakis E. Visuoperceptual speed of karate practitioners at three levels of skill. Percept Mot Skills 1998; $87 \quad$ (1): 96-98. https://doi.org/10.2466/pms.1998.87.1.96

(23) Boonstra AM, Schiphorst Preuper HR, Balk GA, Stewart RE. Cut-off points for mild, moderate, and severe pain on the visual analogue scale for pain in patients with chronic musculoskeletal pain. Pain 2014; 155 (12): 2545-2550. https://doi.org/10.1016/i.pain.2014.09.014

(24) Thornton C, Sheffield D, Baird A. A longitudinal exploration of pain tolerance and participation in contact sports. Scand J Pain 2017; 16: 36-44. https://doi.org/10.1016/..sjpain.2017.02.007

(25) Koropanovski N, Berjan B, Bozic PR, Pazin N, Sanader A, Jovanovic S, et al. Anthropometric and physical performance profiles of elite karate kumite and kata competitors. J Hum Kinet 2011; 30: 107-114. https://doi.org/10.2478/v10078-011-0078-x

(26) Pozo J, Bastien G, Dierick F. Execution time, kinetics, and kinematics of the mae-geri kick: comparison of national and international standard karate athletes. J Sports Sci 2011; 29 (14): 1553-1561. https://doi.org/10.1080/02640414.2011.605164

(27) Koh J. Prevalence rate of chronic overuse pain in taekwondo athletes. J Sports Med Phys Fitness 2017; 57 (10): 1330-1337. https://doi.org/10.23736/s00224707.16.06531-2

(28) Solis-Hernández JL, Rojano-Mejía D, Marmolejo-Mendoza M. Disfuncionalidad de rodilla en la población general y factores asociados. Cir Cir 2016; 84 (3): 208-212. https://doi.org/10.1016/i.circir.2015.10.003

(29) Pop T, Czarny W, Glista J, Skrzypiec M. Influence of traditional karate training on the stability and symmetry of the load on lower limbs. Arch Budo 2013; 9 (1): 39-49. https://doi.org/10.12659/AOB.883840

(30) Langley PC, Ruiz-Iban MA, Molina JT, De Andres J, Castellón JRG. The prevalence, correlates and treatment of pain in Spain. J Med Econ 2011; 14 (3): 367-380. https://doi.org/10.3111/13696998.2011.583303

Número de citas totales / Total references: 30 (100\%)

Número de citas propias de la revista / Journal's own references: 1 (3,33\%)

Rev.int.med.cienc.act.fís.deporte - vol. 20 - número 78 - ISSN: 1577-0354 